Editorial

\title{
Keyhole Neurosurgery: Primum non nocere
}

\author{
Harjinder S. Bhatoe ${ }^{1}$ \\ ${ }^{1}$ Department of Neurosciences, Max Super Specialty Hospital, \\ New Delhi, India \\ Indian J Neurosurg 2015;4:129-131.
}

Medical intervention must primarily aim to be as nontraumatizing as possible. Otherwise the effect of treatment may be worse than the natural course of the disease itself.

-Hippocrates

Neurosurgeons have endeavored to make surgery safe for the patient ever since the inception of neurosurgery as a specialty. The limited capacity of the neural tissue to withstand sustained retraction coupled with rigid covering of the brain has been the major limitation in brain surgery. Besides the lesion itself, the surgical approach to it often results in poor outcome due to brain retraction or transgression of neural tissue. Brain, by virtue of its complex neurophysiology and neurochemical responses to injury, lends itself very poorly to retraction. Excessive intraoperative retraction can lead to cerebral infarction due to increased local cerebral pressure, diminished cerebral blood flow, sacrifice of bridging veins, and so on. The evolution of neurosurgery as a specialty has seen neurosurgeons devising methods to circumvent the need for brain retraction to minimize retraction and to combat the ill effects of retraction. In approaching the lesions of the anterior skull base, many different approaches have been described. Nearly all of them sacrifice large portions of the skull (to be later reconstituted as parts of a jigsaw puzzle), and thus expose large area of the brain, so as to minimize retraction. The other impediment was the absence of adequate illumination of the operative field, resulting in large craniotomies and much approach-related morbidity. This led to evolution of operative neurosurgery in the form of large craniotomies, even for small pathologies. Introduction of operating microscope was a landmark event to improve visualization with magnification. With pioneering works of Albert Rhoton Jr. and M. G. Yasargil, there was renewed understanding of anatomy and surgical approaches. The past two decades have witnessed an explosion in therapeutic technology, and neurosurgery has been in the forefront of devising and adopting newer technologies. The ambition of any neurosurgeon has been to achieve maximum therapeutic effect with least iatrogenic injury, and keyhole surgery has been a major advancement in achieving this goal.

\section{The Keyhole Concept}

The past two decades have witnessed exponential growth of technology in neurosurgery. The net result has been development and refinement in microneurosurgery, neuroendoscopy, and progressive reduction in the invasiveness of neurosurgical procedures. The expectation to achieve maximum therapeutic effect with nil iatrogenic complication is higher than ever before. Growth of neuroimaging coupled with ability to visualize the deepest areas of brain have added new dimension to the neurosurgical technique-the keyhole concept. Tissue trauma is a part of any surgical procedure, and reduction of approach-related morbidity is to be expected with the making of small, precise opening to tackle the lesion. The best way to preserve intact structures is not to touch them and better not to expose them. ${ }^{1-3}$ Unnecessary exposure of the brain to non-physiological environment (viz., room air, cooling, humidity, etc.) should be avoided.

Cranial base techniques are unique for the excellent view they offer to the deep-seated lesion. However, with refinements in techniques, and availability of bright illumination at the site of pathology (by microscope or endoscope), it may not be necessary to resect wide portions of the skull, and attention can be focused on the compartment concerned (-Figs. 1-3). Selecting short, direct, and precise routes to lesions without the manipulation and exposure of unaffected areas is essential for keyhole surgery; undoubtedly, such surgery will qualify for the term "human bio-ecology." 4 Perneczky's keyhole concept, ${ }^{1}$ as a part of the concept bio-ecological neurosurgery, "includes a number of additional features
Address for correspondence Brig. Dr. Harjinder S. Bhatoe, MCh, Director Neurosciences, Max Super Specialty Hospital PPG, New Delhi 110092, India (e-mail: hsbhatoe@gmail.com).
DOI http://dx.doi.org/ 10.1055/s-0035-1570400. ISSN 2277-954X.
(C) 2015 Neurological Surgeons' Society of India
License terms

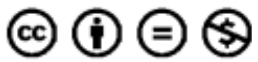




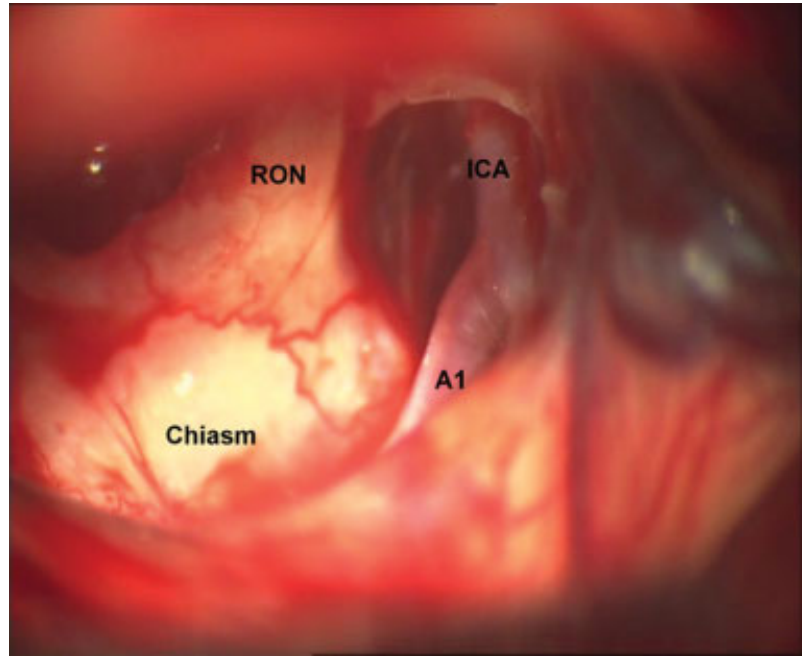

Fig. 1 View after excision of clinoidal meningioma: exposure of optic chiasm and surrounding structures. A1, anterior cerebral artery; ICA, internal carotid artery; RON, right optic nerve.

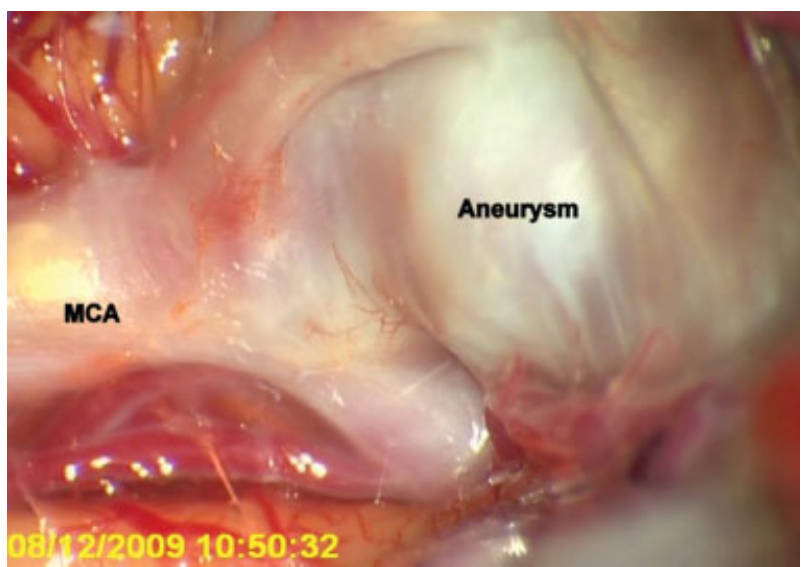

Fig. 2 View of right middle cerebral artery aneurysm (bifurcation aneurysm) by transciliary supraorbital keyhole approach, after splitting of Sylvian fissure, just prior to clipping. MCA, middle cerebral artery.

that provide modern microsurgery with the advantage of reduced traumatisation and, at least, equal effectiveness to remove the lesions as compared with "standard" microsurgical procedures." The term keyhole should be understood in its proper context, that the site chosen literally opens the anatomic compartment of interest like a keyhole. The keyhole concept is defined by the following two principles:

1. The intracranial optical field widens with increasing distance from the skull opening. The natural premise is that the field has been well exposed, brain relaxed, and the passage to the region of interest is clear.

2. Contralateral structures are well visualized.

Therefore, lesions close to the surface of the brain require an approach that is at least as large as the lesion itself. On the other hand, lesions at the base of the brain can be

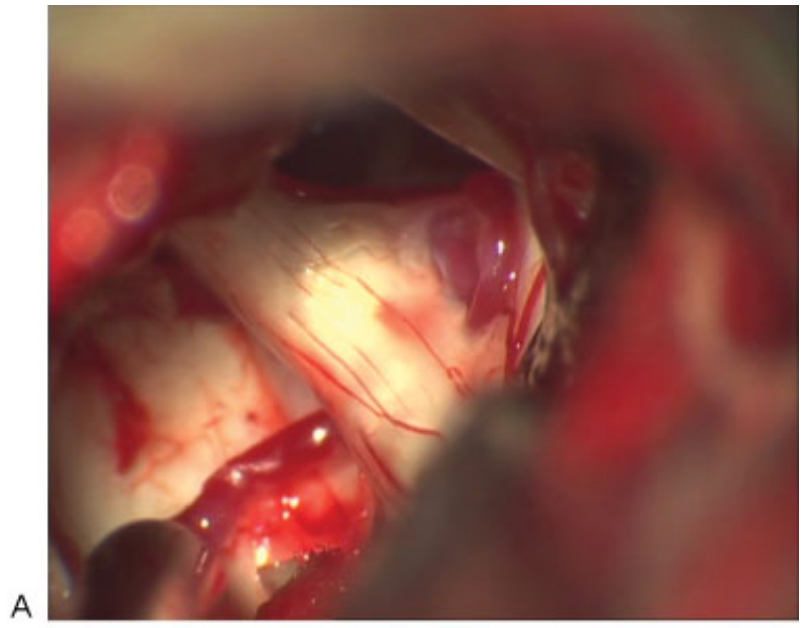

B

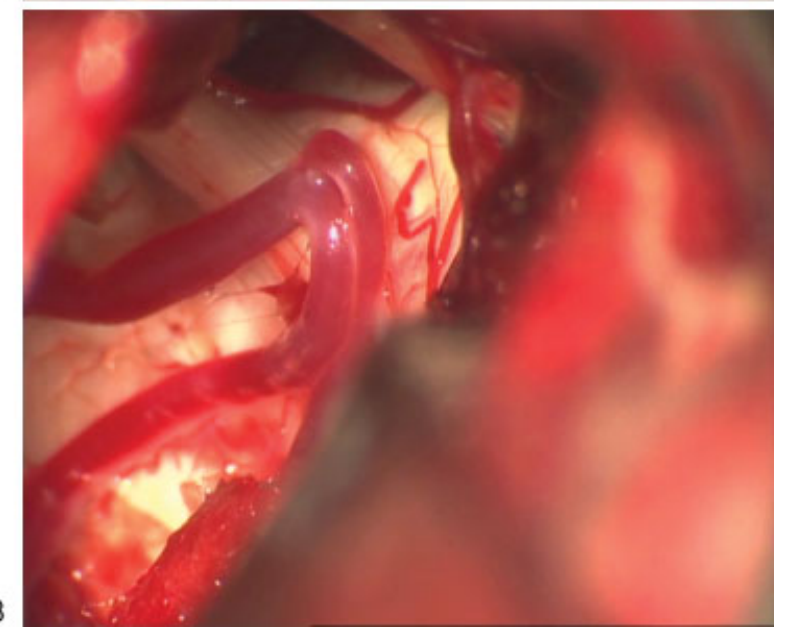

Fig. 3 (A) View of ectatic right superior cerebellar artery by retrosigmoid keyhole approach, densely embedded in the trigeminal nerve, in a patient with trigeminal neuralgia. (B) The artery mobilized from its region of trigeminal nerve compression, near completion of microvascular decompression.

visualized through a very small opening. More than the visualization, it is the size of the instruments required and available that determines the size of the opening.

It is important that the expression "keyhole" be understood as a description of the opening to unlock the exposure of structure(s) of interest, rather than the actual size of the opening, the objective being minimal brain retraction. It is thus, the result of philosophy of minimal invasiveness rather than goal of a small-sized craniotomy. Integration with neuroendoscopy can augment visibility. The obvious advantage of keyhole approach over neuroendoscopy is three-dimensional visualization, familiarity with microanatomy, ability to use both hands, and a shorter learning curve as compared with neuroendoscopy.

Keyhole surgery marks the next step in the philosophy of minimizing approach-related morbidity. The aim of "minimalism" is to minimize the approach-related morbidity. There is a learning curve, and it must be understood that minimalism in today's context follows a training in conventional surgery. Knowledge of subarachnoid spaces and intraventricular topographical 
anatomy is the bedrock of minimally access neurosurgery. Thus, the complications in minimal access neurosurgery often arise from the very concept of approach-related morbidity, and the approach may result in complications that it seeks to avoid. The surgeon is thus the most important variable in the outcome of keyhole surgery. The approach is uniaxial and requires frequent repositioning of microscope. The surgeon must be comfortable working through narrow spaces. The complications can be related to the pathology (attempted removal of a large multicompartmental tumor); the state of the brain (cerebral edema, vascular congestion); inability to slacken the brain by cerebrospinal fluid (CSF) drainage; and early retractor injury perpetuating edema. Refractory brain swelling can prevent approach to the pathology. These complications can result in suboptimal surgery (e.g., incomplete tumor removal and suboptimal clip application) and injury to critical structures (viz., perforators, optic/olfactory apparatus, hypothalamus, eloquent cortex, etc.). Events like intraoperative hemorrhage can cause additional damage as one tries to achieve hemostasis through narrow opening and critical time span. Therefore, one has to anticipate and prevent these events by following the steps of surgery in a well thoughtout sequence. CSF drainage and slackening of brain prevent most of these complications.

\section{References}

1 Perneczky A, Muller-Forell W, van Lindert E, Fries G. Keyhole Concept in Neurosurgery. Stuttgart, Germany; New York, NY: Thieme; 1999

2 Reisch R, Perneczky A. Ten-year experience with the supraorbital subfrontal approach through an eyebrow skin incision. Neurosurgery 2005;57(4, Suppl):242-255, discussion 242-255

3 Reisch R, Perneczky A, Filippi R. Surgical technique of the supraorbital key-hole craniotomy. Surg Neurol 2003;59(3): 223-227

4 Jho HD, Alfieri A. Endoscopic removal of third ventricular tumors: a technical note. Minim Invasive Neurosurg 2002; 45(2):114-119 